\title{
dx.doi.org/10.17488/RMIB.39.2.3
}

\section{Incubadora neonatal con ventilación mecánica embebida}

\section{Mechanical Ventilation embedded in Neonatal Incubator}

\author{
C. A. Mugruza-Vassallo1,3, C. B. Castillón-Lévano², H. Román-Linares² \\ 'Universidad Tecnológica de Lima Sur (UNTELS) \\ ${ }^{2}$ Pontificia Universidad Católica del Perú \\ ${ }^{3}$ Anteriormente en la Pontificia Universidad Católica del Perú
}

\section{RESUMEN}

Se presenta un equipo de soporte de vida neonatal (ESVIN) que emplea la terapia térmica y terapia ventilatoria (neumática) en un solo equipo para proveer ventilación pulmonar con aire caliente, humedecido y enriquecido con oxígeno, en un ambiente caliente, humidificado y estéril. El equipo es capaz de simultáneamente dar ventilación pulmonar e incubar, siendo una de sus características principales la minimización de la condensación del agua en el corrugado y ofrece la característica adicional de evitar la movilización y/o desconexión del neonato para realizar ciertos procedimientos tales como: cirugías e intubaciones, entre otras. Los principales resultados son el tiempo de acceso al neonato menor a $2 \mathrm{~s}$ y minimización de la condensación de agua. Asimismo, los resultados del control térmico son de tiempo de estabilización en el habitáculo de 75 minutos para la temperatura de $36^{\circ} \mathrm{C}$ y tiempo de estabilización de la temperatura de la piel del neonato de 58 minutos.

PALABRAS CLAVE: equipo de soporte de vida neonatal; ventilación pulmonar; incubadora; Presión Inspiratoria Máxima (PIM); Unidad de Cuidados Intensivo Neonatal (NICU) 


\section{ABSTRACT}

A neonatal life support equipment (ESVIN) employing simultaneously thermal therapy and ventilatory (pneumatic) therapy is presented in a single kit to provide pulmonary ventilation with warm, moistened and oxygen enriched air in a warm, humidified and sterile environment. The invention behind ESVIN provided simultaneously pulmonary ventilation and incubation having optimized the minimization of water condensation in the corrugated pipe and offered the additional feature of avoiding the mobilization and / or disconnection of the neonate to perform certain procedures such as: surgeries and intubations, among others. ESVIN has an access to newborns of less than $2 \mathrm{~s}$ and non-visible water condensation. The main results in thermal control were a stabilization time in the newborn compartment of 75 minutes for the temperature of $36^{\circ} \mathrm{C}$ and a stabilization of the temperature of the skin of the neonate of 58 minutes.

KEYWORDS: neonatal life support team (ESVIN), pulmonary ventilation, incubator, Maximum Inspiratory Pressure (PIM), Neonatal Intensive Care Unit (NICU)

\section{Correspondencia}

DESTINATARIO: Carlos Andrés Mugruza-Vassallo INSTITUCIÓN: Universidad Tecnológica de Lima Sur (UNTELS)

DIRECCIÓN: Sector 3 Grupo 1A 03, Av. Central y Av. Bolivar, Villa El Salvador, Perú CORREO ELECTRÓNICO: cmugruza@yahoo.com

\section{Fecha de recepción:}

6 de octubre de 2017

Fecha de aceptación:

30 de enero de 2018 


\section{INTRODUCCIÓN}

En la actualidad, la atención de salud de los neonatos críticos en la Unidad de Cuidados Intensivos Neonatales (UCIN) si bien es de rutina al mezclarse con otros aparatos, es caótica e ineficiente debido a que cada uno de los principales equipos de soporte de vida han evolucionado de forma independiente. Así, se han generado consecuencias negativas tanto para UCIN como para la atención de salud de los neonatos tales como dificultad para manejo, exceso de interfaces entre los equipos y el neonato crítico, interferencia entre equipos, excesivo espacio ocupado por los equipos, exceso de gastos por concepto de adquisición, instalación, mantenimiento de equipos complementarios y la capacitación de profesionales para el manejo de equipos. Este grupo de observaciones se ha realizado por el Grupo de Investigación y Desarrollo de Equipos Médicos y Sistemas (GIDEMS) en 15 años de trabajo, por ejemplo en ${ }^{[1]}$ se consideró un árbol de problemas que incluye la instrumentación y las capacidades y conocimientos en ciencia y tecnología de ventilación mecánica como una de las características base.

Además de los problemas expuestos, hay otros que reportan la mayoría de médicos neonatólogo, como: condensación de agua en los tubos corrugados por donde se administra la mezcla de aire y oxígeno; deficiente distribución de calor en el habitáculo neonatal; dificultad para atender al neonato en caso de emergencia (e.g. paro cardiaco o paro respiratorio)|; y dificultad para volver a conectar catéteres, sondas, tubos, sensores, etc. después de haber pasado la emergencia siendo así que el tiempo de apertura de los equipos para accesar al neonato es de 15 a 18 segundos para una persona entrenada (comunicación personal con neonatólogos, e.g. la neonatóloga Martina Leonila Zegarra Linares).

Para mejorar la atención de los neonatos de mediano riesgo en los hospitales, el GIDEMS de la Pontificia Universidad Católica del Perú (PUCP) desarrolló tres patentes. La primera fue la Burbuja Artificial Neonatal
(BAN), patentada en USA (US6884211) [2] y Perú (PE000622-2002/OIN) [3], la cual está constituida por un circuito cerrado de aire temperado, y por un circuito ventilatorio continuo y ha demostrado tener mejores características funcionales que las incubadoras convencionales en: la propagación uniforme de calor, la adaptación a ambientes no controlados de cuidados intensivos, la duración de filtros bacterianos, el menor ruido sonoro en el habitáculo que contiene al recién nacido, menor probabilidad de contaminación entre neonatos, la menor pérdida de calor, el menor consumo de oxígeno, etc. Además, se le han incorporado otras ventajas como: reproductor de sonidos para mejorar el bienestar de los neonatos, mezclador de aire y oxígeno, y mejor sistema de humidificación. Sin embargo, dicha BAN no cuenta con un circuito de ventilación pulmonar para neonatos. Por ello, se realizó la segunda patente que consiste en un sensor de flujo gaseoso bidireccional, patentado en USA (US7028560) ${ }^{[4]}$ y Perú (PE000594- 2002/OIN) cuya idea de la variación de las medidas de acuerdo a la compresión en las tuberías se basaron en el manejo de presiones en tuberías flexibles reportados experimentalmente ${ }^{[1]} \mathrm{y}$ que puede variar de acuerdo al gas ${ }^{[20]}$. Finalmente, la tercera patente que se gestionó fue una Burbuja Neonatal con Presurizador de Vías Aéreas, solicitud de patente PE001492-2007/ OIN ${ }^{[5]}$, el cual posee las ventajas de la BAN (asepsia, ambiente humidificado, temperatura uniforme y bajo ruido sonoro) y le añade una función muy importante para la atención de neonatos de alto riesgo que es el control de parámetros de la mezcla gaseosa que se administra a las fosas nasales del recién nacido crítico como temperatura y presión positiva. También incluye un sensor de flujo gaseoso bidireccional desarrollado por el GIDEMS. Sin embargo, este prototipo si bien presuriza las vías aéreas del neonato, no controla el flujo de la ventilación pulmonar del neonato.

Por otro lado: la patente EP1529547 presenta un equipo que difiere de los ventiladores convencionales en su portabilidad, funcionando complementaria- 
mente con las incubadoras ${ }^{[6]}$, la patente de invención DE102006030520 de la empresa Dragar ha desarrollado un sistema de control para ventilación pulmonar que difiere de los demás solamente en su algoritmo de control, y que también funciona complementariamente con incubadoras ${ }^{[7]}$. Asimismo, las solicitudes de patentes US2008125619 ${ }^{[8]}$, US2008081943 ${ }^{[9]}$ y US2008076962 ${ }^{[10]}$ muestran los últimos inventos sobre incubadoras neonatales, representando las últimas modificaciones importantes al estado de la técnica, que tampoco integran la función de ventilación pulmonar.

Todas estas invenciones no integraban la función de ventilación pulmonar con la función de incubación. Además, todas ellas implican la movilización del neonato para realizar ciertos procedimientos tales como cirugías e intubaciones, entre otras.

Para la atención de neonatos de alto riesgo se emplea la Unidad de Cuidados Intensivos Neonatal (NICU), que de acuerdo a una estadística de 229 centros en EE. UU y 290 a 349 centros de Reino Unido, que suele cobijar a los recién nacidos entre $500 \mathrm{~g}$ y $1500 \mathrm{~g}$ entre un rango de 40 a 90 días como reportó Merritt [11]. Se sabía que neonatos de menos de $2500 \mathrm{~g}$ al nacer son 50 \% más propensos que los de peso normal a requerir de educación especial según Chaiking y Corman ${ }^{[12]}$. Existe evidencia que, para neonatos de menos de 30 semanas de gestación o menos de $1250 \mathrm{~g}$, un menor tiempo de ventilación pulmonar favorece el incremento del tamaño cerebral a los 2 años como reportó Anderson ${ }^{[13]}$ lo cual se toma en este estudio como un índice de la calidad de ventilación en una NICU. Es decir, si el ambiente de la NICU es mejor, el neonato necesitará un menor tiempo de terapia de ventilación pulmonar.

Castillón Lévano, Mugruza Vassallo y Coello Durand plantearon una solución presentada y publicada por el Tratado de Cooperación de Patentes (PCT) como "EQUIPO PARA ATENCIÓN INTEGRAL DE NEONATOS CRÍTICOS” en 17 de marzo de 2011 (WO/2011/030177)
${ }^{\text {[14] } q u e ~ a c a ́ ~ l l a m a m o s ~ e q u i p o ~ d e ~ s o p o r t e ~ d e ~ v i d a ~ n e o n a-~}$ tal (ESVIN). ESVIN consistió en reivindicar un equipo para atención neonatal que integre las funciones de un ventilador pulmonar, incubadora, mezclador de gases, humidificador y monitor de signos vitales para que este único equipo tenga la capacidad de ventilar los pulmones con aire calentado humedecido y enriquecido con oxígeno, en un ambiente temperado humidificado y estéril, pero que evite la condensación del agua en el corrugado de administración de la mezcla gaseosa para ventilación, facilite la atención de emergencia y evite la excesiva manipulación del neonato durante la atención y la función de supervisión de signos vitales.

De esta manera en este trabajo se buscó materializar la construcción y empleo biomédico de este concepto de ventilación mecánica sin condensación en una incubadora neonatal mediante pruebas neumáticas de ventilación, térmicas en la piel del neonato y fisiológicas en ensayos en pulmones animales.

\section{METODOLOGÍA}

\section{Concepto del equipo de soporte de vida neonatal}

Para el desarrollo del Equipo de Soporte de Vida Neonatal se han tomado en cuenta las normas: IEC 60601-1-4 ${ }^{[15]}$, que da recomendaciones sobre la seguridad para la fabricación; IEC 60601-2-12 (2001) ${ }^{[16]}$, que especifica los requerimientos para realizar ventilación pulmonar, y IEC 601-2-19 [17], que establece los requerimientos exigibles para el desarrollo de incubadoras con temperaturas de mando de 32 a $36^{\circ} \mathrm{C}$ en períodos mayores a 1 hora y cuya temperatura media dentro de la incubadora no debe diferir en más de 0,8 ㄷ․

Asimismo, para el diseño del prototipo se utilizó la metodología "Modelo en V" (e.g. en los 1990s ${ }^{[18]}$ y la década de $2000{ }^{[19]}$ ), que consiste en dividir el sistema en subsistemas, y los subsistemas en otras divisiones de nivel inferior y así sucesivamente. 
Tomando en consideración que el manejo del flujo a bajas presiones permite cuantificar una relación entre la presión y el flujo por medio de la ecuación de Van der Waals para gases ideales ${ }^{[20]}$, en la Figura 1.a se presenta un esquema del funcionamiento del sistema eléctrico-neumático del flujo ventilatorio del equipo presentado en la solicitud de patente $\mathrm{WO} / 2011 / 030177^{[17]}$ que comprende un primer recipiente con agua (308) que limita tanto la presión de inspiración como la presión de espiración del neonato, y un segundo recipiente con agua (310) que humidifica el gas en la línea de inspiración, que en conjunto con los demás elementos indicado en la Figura 1 funciona de la siguiente manera: se puede controlar y visualizar la presión inspiratoria y específicamente la presión pico (ver Figura 1.a parte superior), para la fase inspiratoria, la primera tubería (309) sumergida en el primer recipiente con agua (308) muestra visualmente que la inspiración no excede la presión manométrica inspiratoria máxima y si la presión supera esa presión inspiratoria máxima hay fuga de aire en dicha tubería, limitando la presión positiva de la línea de inspiración; y la segunda tubería (317), sumergida en el primer recipiente con agua (308), asegura la presión manométrica positiva al final de la espiración que por ejemplo se presenta en torno a $1 \mathrm{cmH} 2 \mathrm{O}$ según se observa más adelante en la Figura 7. Este manejo de bajos cambios de presión y con pocos niveles de flujo de volumétrico del gas en condiciones similares a la ventilación mecánica se observó en simulaciones previas ${ }^{[20]}$, pero recién en la patente en mención y en este trabajo se observó experimentalmente.

El concepto del equipo de soporte de vida neonatal se basa en un sistema neumático controlado por un sistema electrónico tal como se puede apreciar en la Figura 1.b, donde: el compresor (7) es el elemento que moviliza y suministra el flujo regulado de aire-oxígeno que se entrega al neonato localizado en el habitáculo neonatal (10); el control de todo el sistema neumático y la temperatura del anillo térmico (11) son a)

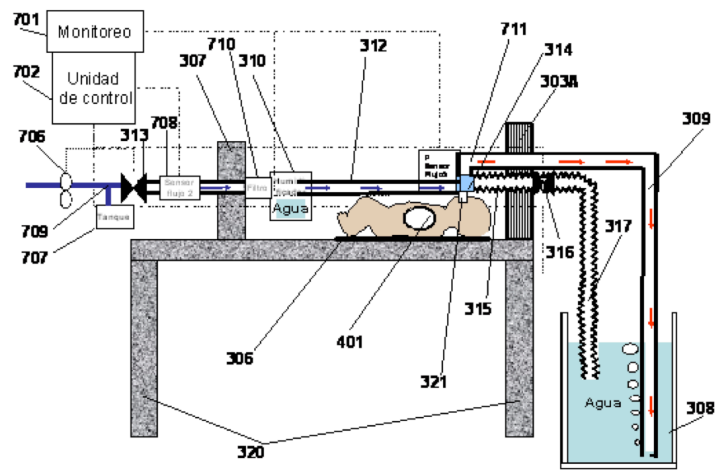

b)

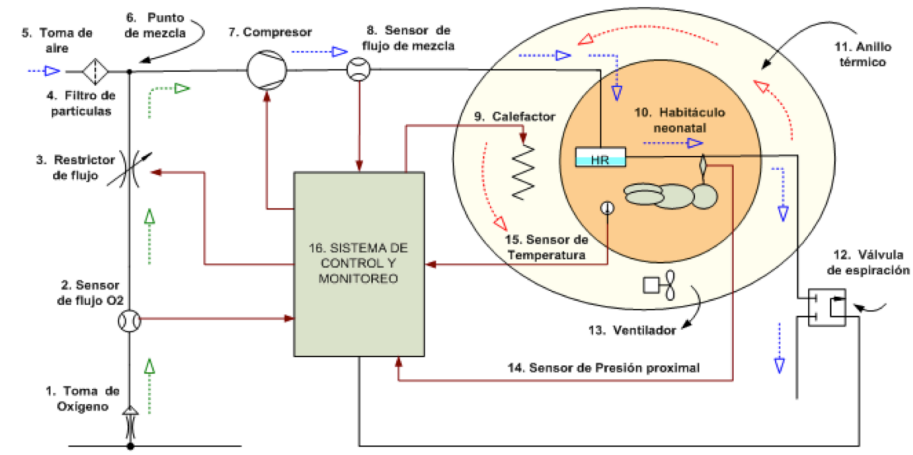

Inspiración con sobrepresión evitada con la fuga de gas por medio de (309)

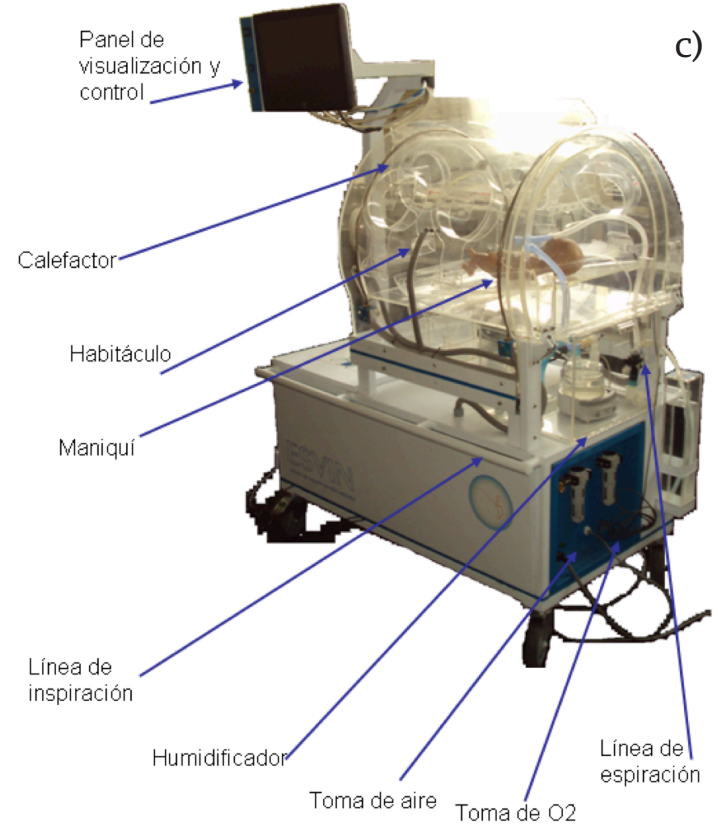

FIGURA 1. Esquema del concepto del equipo de soporte de vida neonatal. (A) Diseño general en solicitud de patente publicada WO/2011/030177 ${ }^{[17]}$ (B) Diagrama conceptual (C) Fotografía de la implementación final. 
realizados por el sistema de control y monitoreo (16); la válvula de espiración es el elemento que controla tanto el nivel de presión del gas que entrega al recién nacido y así como el tiempo que dura cada ciclo respiratorio ${ }^{[14]}$. Así la válvula también es controlada por el sistema de control y monitoreo (16) en las tomas de regulación de presión conectados en serie a las tomas de aire y oxígeno en la Figura 1.c

La implementación final consiste en un sistema neumático y térmico controlado por un sistema electrónico tal como se puede apreciar en la Figura 1.c, donde se muestra: el panel de visualización y control, los calefactores, el habitáculo, un maniquí o pulmón que es objetivo de este trabajo. De esta manera, se simula el neonato, el tubo de inspiración, el humidificador, la toma de aire y de oxígeno (O2) y el tubo de espiración.

En la Figura 2 se ilustra el funcionamiento del sistema mecánico del equipo, donde la ventaja técnica y funcional está en acceso rápido al neonato mediante el desplazamiento del anillo cilíndrico térmico a lo largo

DISEÑO MECÁNICO EN PATENTE IB2009007014

a)

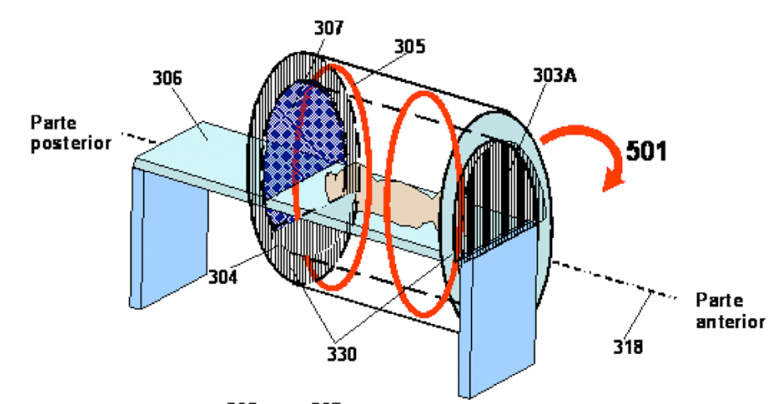

b)

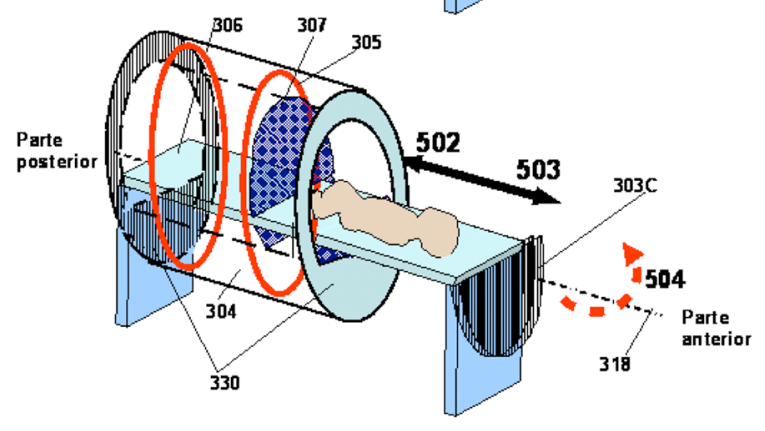

del eje de anillo (318) sin necesidad de mover al neonato, esta función permite al personal de salud realizar procedimientos de emergencia sobre el neonato que está en terapia ventilatoria y térmica. En la Figura 2.a, en el gráfico superior se muestra el anillo térmico cerrado en su parte posterior por una puerta, permitiendo así el funcionamiento adecuado de la terapia térmica, además se cuenta con una puerta anterior la cual se puede abrir (501) desde su posición superior (303A) hacia su posición inferior (303C). En la Figura 2.b, en el gráfico inferior se muestra la puerta desplegada y el anillo térmico desplazado que permite acceso al neonato, a la que se accede cuando la puerta giró (501) a su posición inferior (303C), y se desplaza (502) el anillo térmico (304) que son un bloque mecánico según se observa en las flechas que indican el movimiento 502 y $503^{[14]}$. En la implementación física de forma similar el diseño final muestra en las partes Figura 2.d y 2.e como se realiza el desplazamiento mecánico del cuerpo con el anillo término y el giro de la puerta que permite un acceso inmediato al neonato desde la posición inicial (Figura 2.a).

\section{IMPLEMENTACIÓN EN EQUIPO BIOMÉDICO}

c)

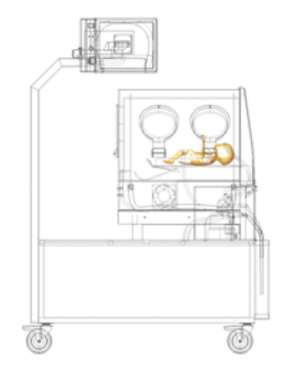

e)

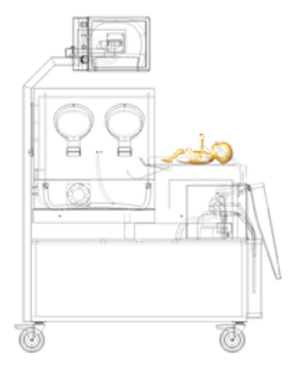

d)

FIGURA 2. Esquema del concepto mecánico del equipo de soporte de vida neonatal. (A, B) Diseño general en solicitud de patente publicada WO/2011/030177 (IB2009007014) ${ }^{[17]}$ (C, D, E) Implementación final. 
En la Figura 3.a se muestra a un ventilador (319) que es fuente del flujo de aire que circula sobre las resistencias calefactoras y que uniformiza la temperatura del flujo del anillo térmico. Figura 3.b, se observan las compuertas (601) con un ángulo que favorece el acceso al neonato, las flechas que indican el flujo del aire que

\section{DISEÑO MECÁNICO EN PATENTE IB2009007014}

a)
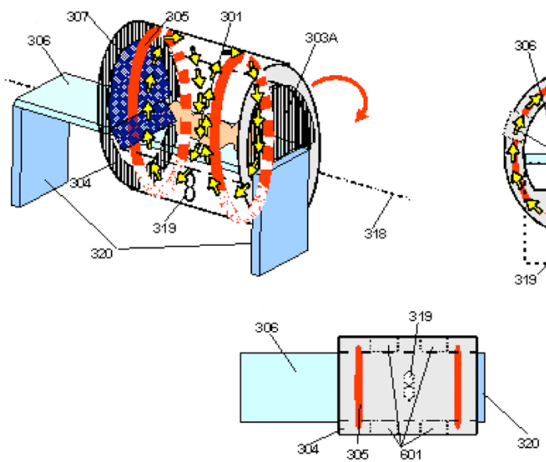

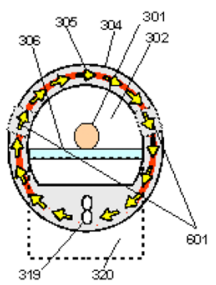

C) es calentado por las resistencias calefactoras. Figura 3.c, se ven compuertas (601) que permiten el acceso al neonato según ${ }^{[14]}$, y en las Figuras 3.d y 3.e se observa lo correspondiente a las correspondientes Figuras 3.a y 3.b tanto cuando hay terapia térmica y/o cuando hay terapia ventilatoria.

IMPLEMENTACIÓN EN EQUIPO BIOMÉDICO

b)

d)

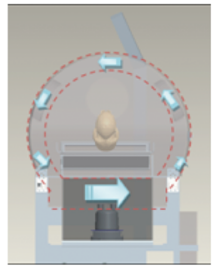

FIGURA 3. Esquema del concepto termoventilatorio del equipo de soporte de vida neonatal. (A, B, C) Diseño general en solicitud de patente publicada WO/2011/030177 (IB2009007014) ${ }^{[14]}$ (D, E) Diseño industrial empleado para la implementación final.

\section{Electrónica}

El diseño e implementación del sistema electrónico está enfocado en la robustez y confiabilidad de su funcionamiento, los bloques básicos que la conforman se ilustran en el Figura 4. Los sensores de temperatura (rango 25 a 37 oC @ 10000 a 6014 ohm @ error lineal de temperatura de -4.39 a $-4.09 \% /{ }^{\circ} \mathrm{C}$, Spectrum $1004 \mathrm{Z}^{[21]}$ ), presión (rango -70 a 70 cmH2O, Honeywell 40PCo01B1A ${ }^{[22])}$ y flujo (rango hasta 20 LPM, cambios de flujo de 5 $\mathrm{LPM} / \mathrm{s}^{[23]}$ ). El sensor de flujo mide $2 \mathrm{LPM}$ en cambios de $0.4 \mathrm{~s}$, que está en el rango de ventilación neonatal y con una relación de I/E de 1/2 permite ventilar y sensar en tiempo real hasta 50 respiraciones por minuto. Las calibraciones de flujo volumétrico fueron basadas en el desarrollo de previos considerando el desplazamiento de fluido incompresible generando diferencias de presión proporcionales al volumen ${ }^{[24]}$ y cuya calibración a compliancia constante ${ }^{[25]}$ ha mostrado ser eficiente en flujos volumétricos del orden neonatal ${ }^{[1]}$.
Cuenta con una unidad primaria de control cuyos componentes principales se describen en la Tabla 1, la cual utiliza los datos que recibe de los sensores de temperatura, presión y flujo para ejecutar rutinas y procedimientos que afectan el estado y magnitud de los actuadores como: calefactor, compresor, ventilador, válvulas y humificador con el fin de mantener constantes los parámetros configurados por el usuario mediante la interfaz de usuario, en dicha interfaz se proyectan las gráficas y datos numéricos de los diferentes parámetros monitoreados de forma local, por medio de un monitor de pantalla táctil y de forma remota a través de una conexión Ethernet. El sistema (ver Figura 4) cuenta con una unidad de respaldo, dicha unidad cuenta con sensores independientes de temperatura y flujo y es capaz de modificar el comportamiento de los actuadores, frente a fallos de la unidad de control primaria. Todo ello con el propósito de proteger la integridad del paciente. 


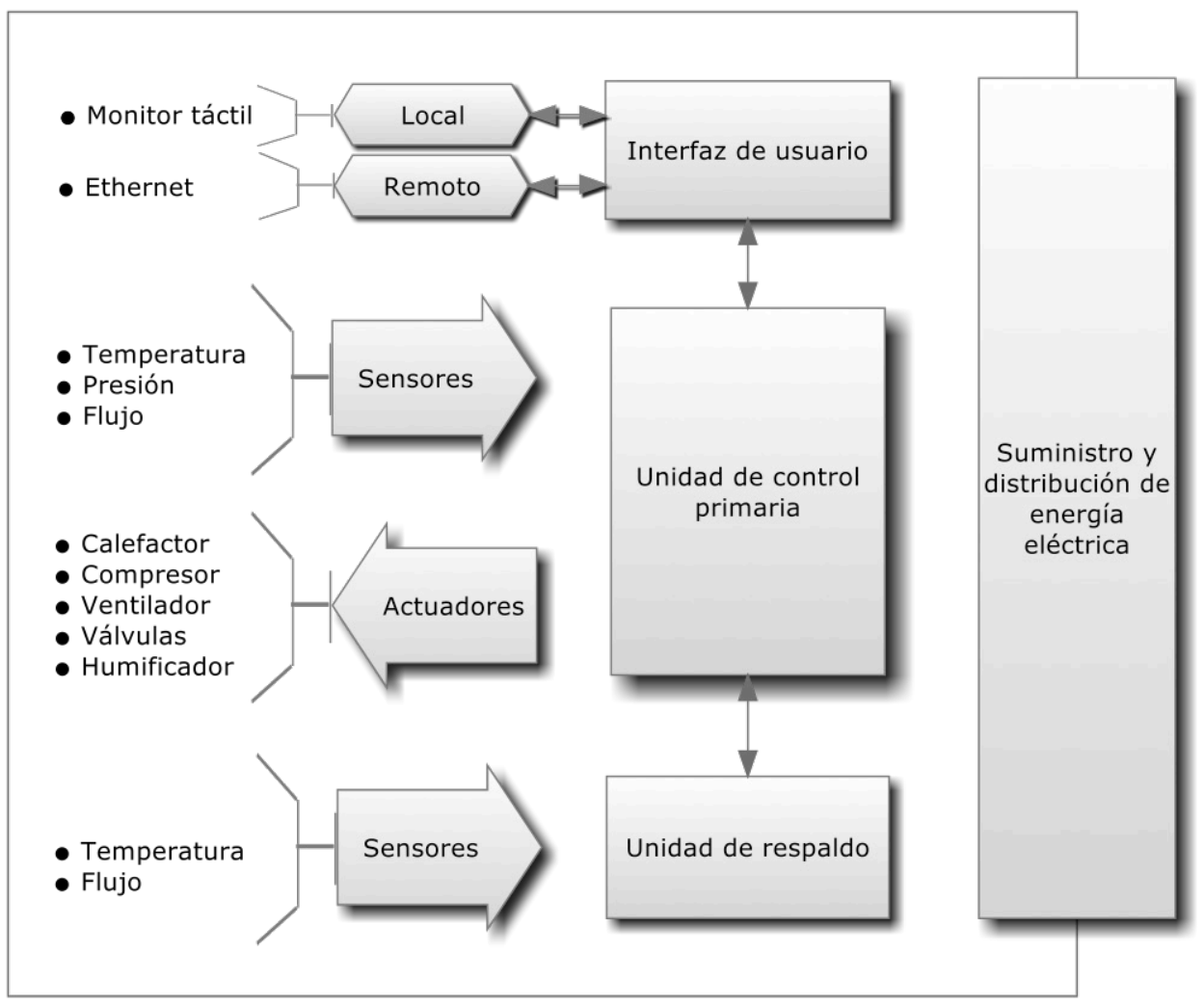

FIGURA 4. Esquema del concepto electrónico del equipo de soporte de vida neonatal.

Finalmente, el suministro y distribución de energía eléctrica proporciona los voltajes y corrientes adecuados para cada componente del sistema electrónico.

\section{TABLA 1. Principales componentes electrónicos de ESVIN.}

\begin{tabular}{|l|l|}
\hline \multicolumn{2}{|c|}{$\begin{array}{c}\text { Principales componentes electrónicos } \\
\text { Relacionados a la unidad primaria }\end{array}$} \\
\hline $\begin{array}{l}\text { Módulo de adquisición de } \\
\text { datos }\end{array}$ & $\begin{array}{l}\text { TSADC16, 16 bits de } \\
\text { resolución y } 100 \mathrm{ksps}\end{array}$ \\
\hline $\begin{array}{l}\text { Módulo de procesamiento } \\
\text { de datos }\end{array}$ & TS-7350, ARM a 200 MHz \\
\hline $\begin{array}{l}\text { Interfaz gráfica con el } \\
\text { usuario }\end{array}$ & ET1515L \\
\hline \multirow{3}{*}{ Sensores } & $\begin{array}{l}\text { Temperatura Spectrum } \\
\text { 1004Z }\end{array}$ \\
\cline { 2 - 3 } & $\begin{array}{l}\text { Presión Honeywell } \\
\text { 40PC001B1A }\end{array}$ \\
\cline { 2 - 2 } & Flujo AWM5104VA \\
\hline
\end{tabular}

\section{Control}

En la Figura 1 se presentó el diseño e implementación del sistema de control de temperatura en el habitáculo neonatal (10) de ESVIN, basado en la norma internacional IEC 60601-2-19 ${ }^{[15]}$ referente a la seguridad básica y funcionamiento de las incubadoras neonatales.

Para encontrar un modelo matemático que describiera el comportamiento dinámico del sistema se usó el Método de Identificación de Sistemas. El resultado permitió diseñar y simular la estrategia de control PID digital según los requerimientos de control de temperatura del habitáculo, de acuerdo a ${ }^{[15]}$. El control PID de 2 polos tuvo parámetros de sintonización originalmente aproximados y finalmente ajustados experimentalmente para una temperatura programada mayor a $35^{\circ} \mathrm{C}$ fueron $z_{1}=0.98$ y $z_{2}=0.9$ para $k=1 \mathrm{y} \mathrm{la}$ constante auxiliar $k T$, ver Ecuación (1)

$$
k T=k /\left(1-z_{1} z_{2}-z_{1}-z_{2}\right)
$$




\section{Ensayos pre-clínicos}

Para el diseño y desarrollo de equipos que serán utilizados en seres humanos, como es el caso de ESVIN, es necesario que, antes de su uso en la práctica médica, atraviesen una serie de pruebas llamadas Ensayos Preclínicos, inicialmente con órganos aislados y luego con animales de experimentación, con la intención de identificar posibles errores y fallas de los sub-sistemas operativos, las cuales serán subsanadas y puestas a punto para garantizar su perfecto funcionamiento.

Primero, según el conocimiento de los presentes autores, no hay tablas reportadas de compliancia a ventilación de presión soporte; por ello, se han buscado gráficos de la literatura y en base a ello se calculó la compliancia pulmonar, para validar la ventilación pulmonar con el ensayo pre-clínico. Dado que generalmente vienen como gráficas en otros artículos científicos, procedimos a realizar una extracción de curvas de las figuras y de allí se calculó la compliancia pulmonar. Los pasos fueron: copiar la figura en un editor gráfico (en nuestro caso Irfanview disponible en www.irfanview.com/), almacenarlo en formato TGA, pasar la figura a escala de grises, de allí hemos extraído las curvas por medio del MatLab y se realiza el cálculo de compliancia dinámica (Cdin) según la Ecuación (2).

$$
C_{\text {din }}=\Delta \text { Volumen } / \Delta \text { Presión }
$$

Segundo, se realizó la disección de pulmones frescos de conejos sacrificados el mismo día del experimento. Para ello, se llevó a cabo una incisión cérvico-toráxica, visualizándose tráquea, laringe y mediastino anterior, posteriormente se procedió a la disección lateral visualizándose ambos pulmones y corazón, los cuales fueron extraídos de manera intacta. Se seleccionaron animales de masas equivalentes a recién nacidos prematuros entre 26 semanas y 32 semanas de gestación aproximadamente, edades que requieren con más frecuencia el uso de asistencia respiratoria mecánica en la práctica clínica.
Los pulmones se colocaron en solución fisiológica (cloruro de Sodio al 0,9\%) y se procedió a intubar la tráquea con tubo endotraqueal $2.5 \mathrm{~mm}$ que se fijó a $1 \mathrm{~cm}$ aproximadamente por encima de la bifurcación de la tráquea con Catgut Crómico 2/0 para evitar fugas de aire.

Finalmente, se corroboró si el nivel de compliancia de los pulmunes de conejo son ventilados adecuadamente y a una compliancia similar a la que se puede deducir de los datos reportados en la literatura.

\section{RESULTADOS}

\section{Resultados del tiempo de apertura del equipo y acceso al neonato}

El tiempo de apertura para el acceso al neonato es menor a 2 segundos, pudiéndose abrir la compuerta (Figura 5.b y 5.c) en simultáneo con el bloque mecánico que contiene los anillos térmicos (Figura 5.d). Tal como se puede apreciar el proceso toma menos de 2 segundos desde que el personal de salud observa el interior de ESVIN (Figura 5.a).

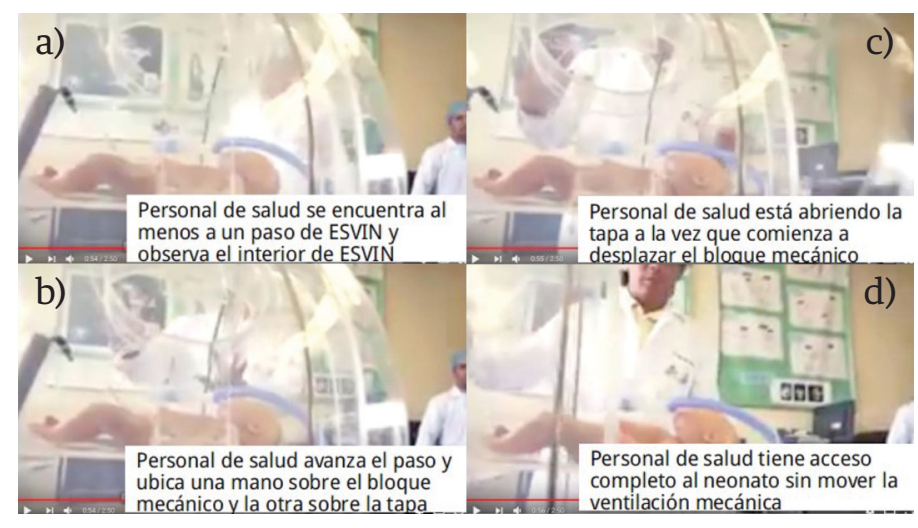

FIGURA 5. Apertura del equipo y acceso al neonato.

En la parte inferior izquierda se muestra los tiempos, resultando el proceso en menos de 2 segundos.

\section{Resultados del lazo de control de temperatura en el habitáculo y piel}

Se comprobó la evolución de la humedad y temperatura del aire en el habitáculo de la incubadora a lo largo de 3 horas. El ensayo realizado, consistió en configurar 
la incubadora encender el sistema de humidificación en el modo aire a $36.0^{\circ} \mathrm{C}$, y con un valor inicial de humedad de $25.0{ }^{\circ} \mathrm{C}$ y luego de unas horas llevarlo a $36.0^{\circ} \mathrm{C}$ presentado en la Figura 6.

En la Figura 6.a y 6.b se observa la respuesta del sistema del control del habitáculo en lazo cerrado sin considerar al simulador del neonato, mientras que en la Figura 6.c y 6.d se observa la respuesta del sistema del control en la piel del neonato. En la Figura 6.a se observa que el tiempo ( $\mathrm{Tr}$ ) en temperatura en que el habitáculo alcanza el valor deseado de $36.0^{\circ} \mathrm{C}$ es de 52 minutos, mientras que en la Figura 6.c el tiempo en que la temperatura de piel del neonato alcanza los 36.0 ${ }^{\circ} \mathrm{C}$ es de 58 minutos.

a)

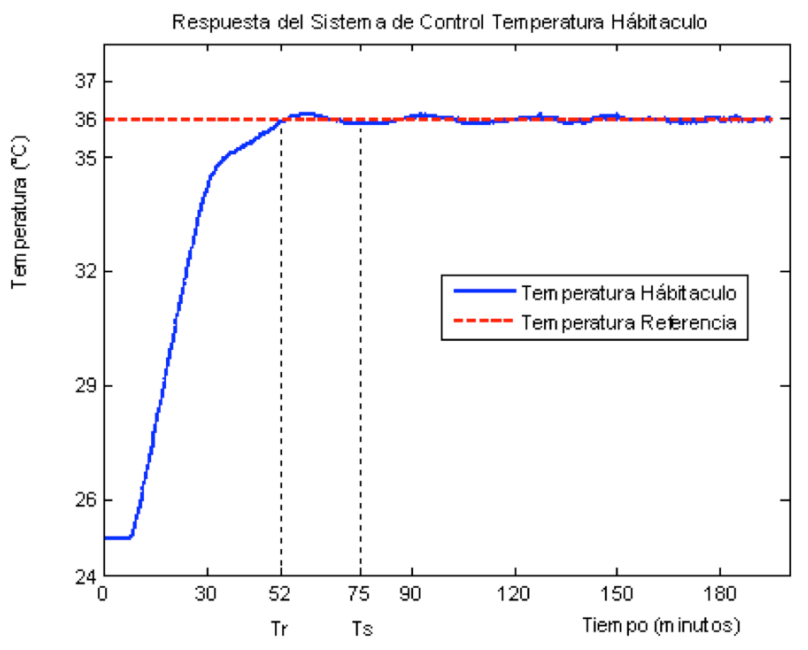

b)

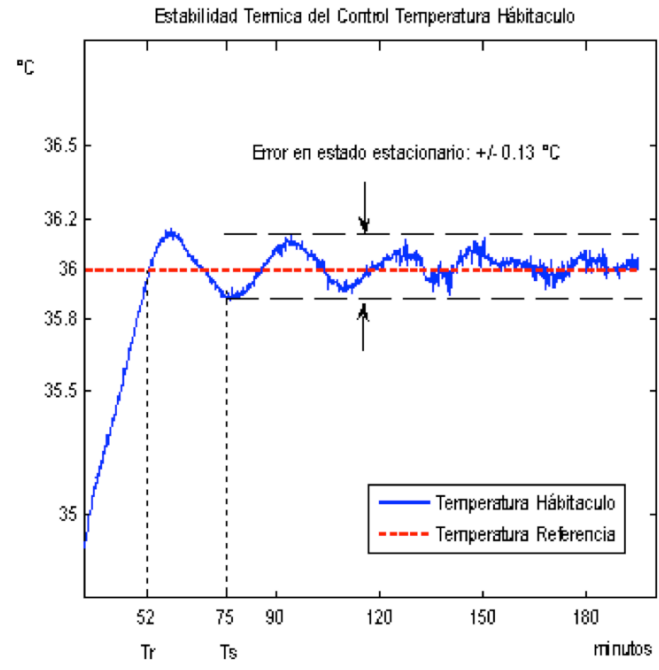

Por otro lado, en la figura 6.b se observa que el error de estado estacionario en temperatura en que el habitáculo alcanza el valor deseado de $36.0^{\circ} \mathrm{C}$ es de 0.13 ${ }^{\circ} \mathrm{C}$, mientras que en la Figura 6.d el tiempo en que la temperatura de piel del neonato alcanza los $36.0^{\circ} \mathrm{C}$ es de $0.09^{\circ} \mathrm{C}$ en un rango de $0.17^{\circ} \mathrm{C}$ de variación máxima.

De esta forma, en este nuevo equipo de soporte de vida neonatal, los resultados del sistema de control permitieron lograr una variación menor a $0.3^{\circ} \mathrm{C}$ cumpliendo la norma y además el tiempo de estabilización fue menor a una hora, solicitado por la norma EC 6012-19 elevando la efectividad y fiabilidad con respecto a los anteriores diseños del GIDEMS y otros reportados en la literatura.

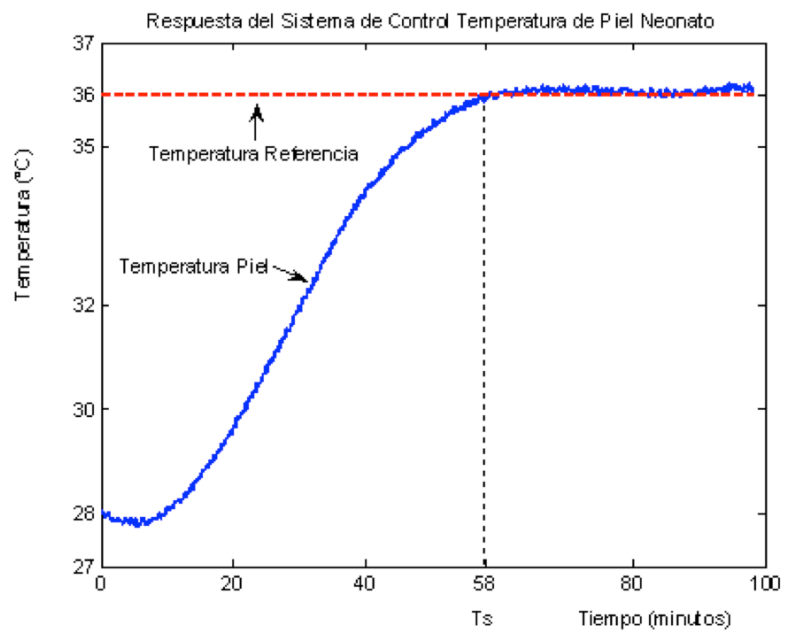

c)

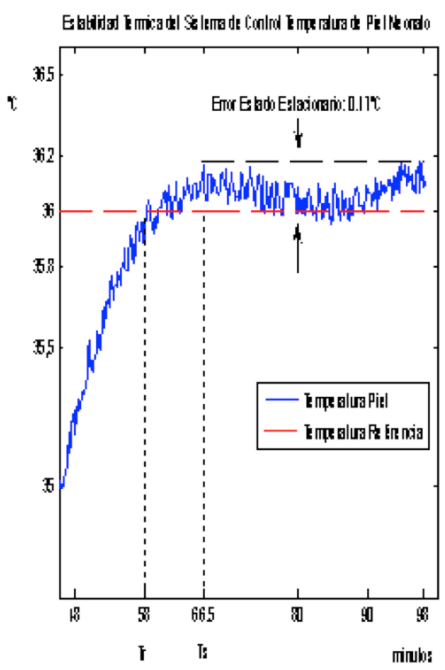

d)

FIGURA 6. Respuesta en lazo cerrado para una temperatura de referencia de $36^{\circ} \mathrm{C}$. 


\section{Resultados de flujo Ventilador Pulmonar}

El ventilador del Prototipo Experimental: Equipo de Soporte de Vida Neonatal (ESVIN), tiene la capacidad de brindar asistencia respiratoria mecánica controlada, con diferentes presiones positivas intermitentes, ciclado por tiempo y limitado por presión y también brinda soporte respiratorio tipo CPAP (Presión Positiva continua en la vía aérea) que es una forma de ventilación no invasiva.
Se obtuvieron gráficas de presión proximal, flujo inspiratorio y volumen medio espirado coherentes con el proceso de ventilación a presión positiva intermitente.

La válvula de seguridad de presión se ha comportado de acuerdo a lo diseñado, mostrando su eficacia.

A continuación, se presenta la ventilación pulmonar en modo sincronizado.

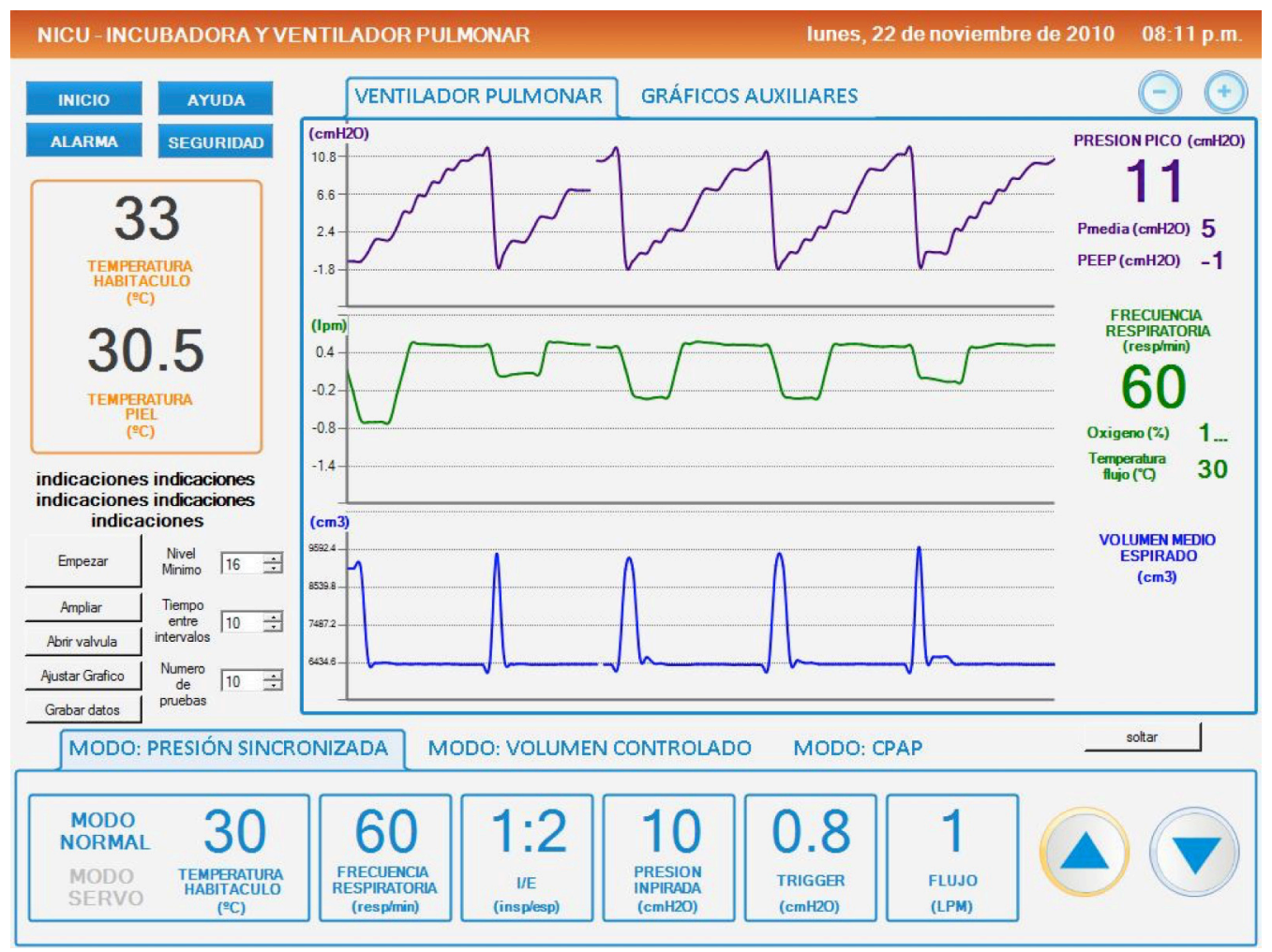

FIGURA 7. Resultado de modo sincronizado mostrados dentro de la interfaz gráfica de usuario del prototipo,

configurado en modo touch screen, en la parte izquierda (recuadro naranja se observa el resultado de la parte térmica y en la parte derecha se observa la ventilación pulmonar.

\section{Resultados Modo Sincronizado}

En la Figura 7 se muestra la gráfica de presión y volumen del modo sincronizado para un flujo de 1 LPM y 60 respiraciones por minuto.

El equipo muestra todas las variables necesarias para que se haga un seguimiento y manejo óptimo de la ventilación pulmonar. En la Figura 7 se muestra la interfaz gráfica de ESVIN. En la parte izquierda se observa los parámetros y monitoreo de la función de incubación; en la parte central se muestran los gráficos de presión proximal, flujo inspiratorio y volumen medio espirado; en la parte derecha se observa los parámetros y medida de ventilación pulmonar: presión pico, presión media, presión positiva al final de la espiración (PEEP), frecuencia respiratoria, porcentaje de oxígeno, temperatura del flujo de inspiración; y en la parte inferior se muestra los parámetros de ventilación del modo selec- 
cionado "presión sincronizada", que muestra la temperatura del habitáculo, la frecuencia respiratoria, la relación inspiración/espiración (I/E), la presión inspirada, el disparo o "trigger" de presión y el flujo volumétrico.

\section{Evaluación pre-clínica}

Siguiendo el primer paso, de la ventilación de presión soporte documentada en la literatura, en la Figura 8.a-d se muestra la ventilación de presión soporte según Becker y Donn ${ }^{[26]}$. Las formas de onda ayudan a distinguir las respiraciones mandatarias (que proveen el soporte total, ver las flechas en Figura 8.a de las respiraciones espontáneas realizada con presión soporte, predeterminadas para liberar la presión soporte. Así, es posible realizar el cálculo de la compliancia pulmonar (Figura 8.d) según (2) para elegir/ corroborar el modelo pulmonar a emplearse en el ensayo pre-clínico.

a)

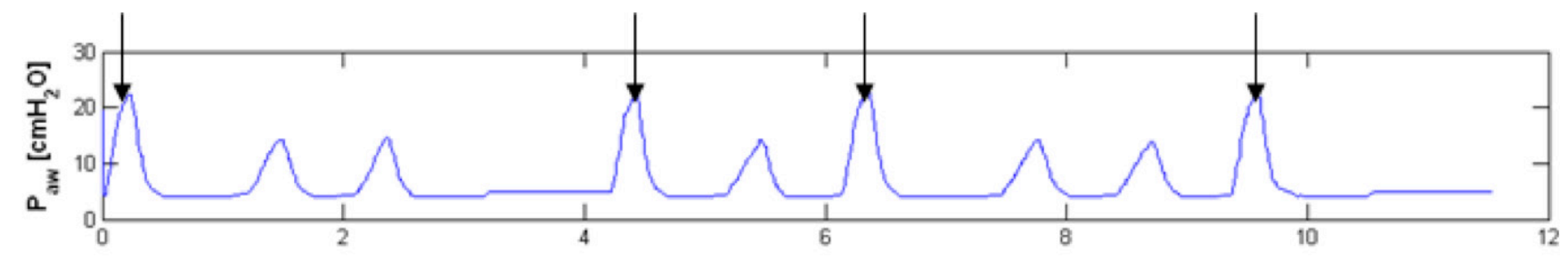

b)

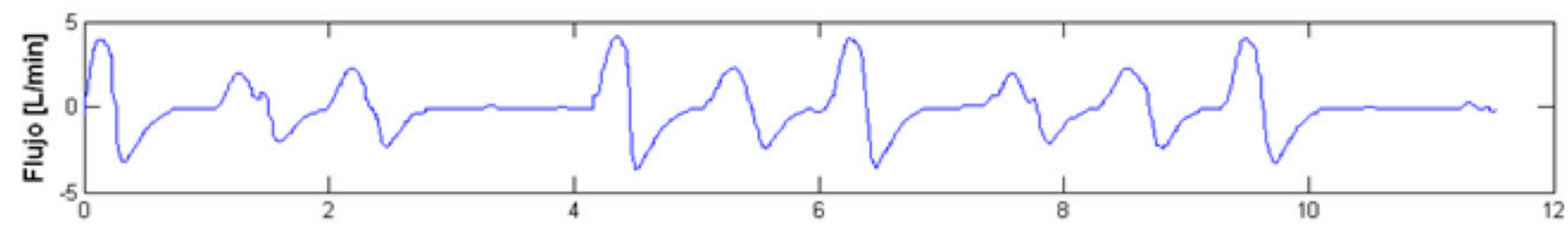

c)

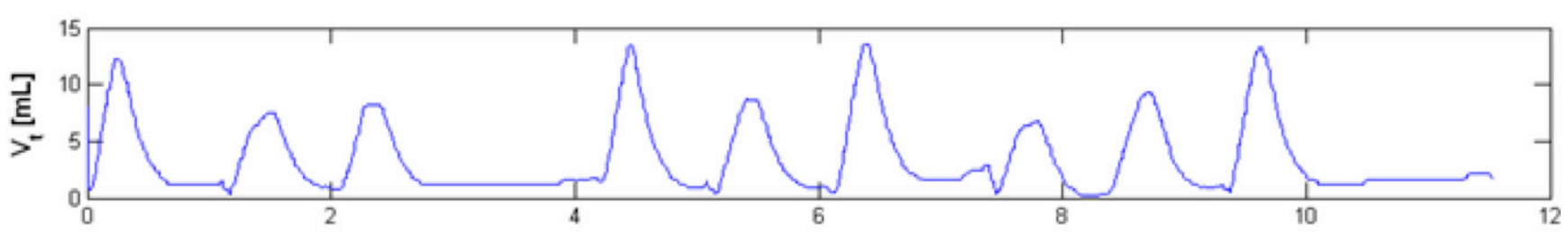

d)

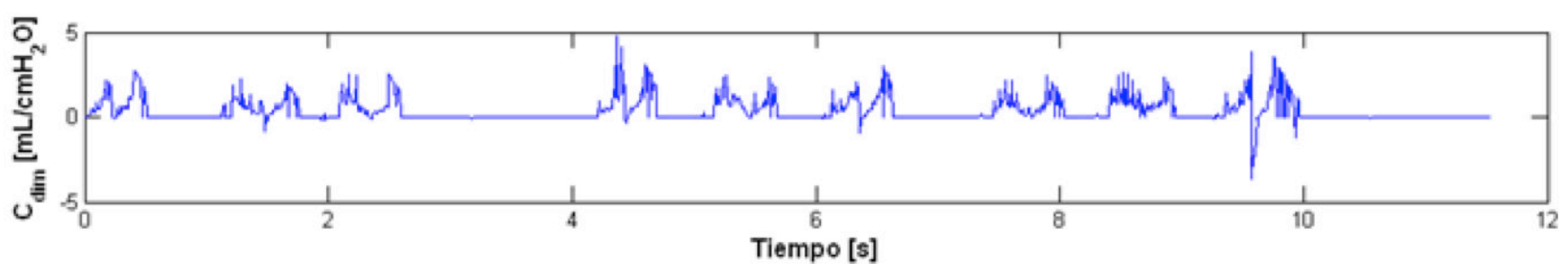

FIGURA 8. Extracción del parámetro de compliancia de presión soporte para emplear dicho rango en los ensayos pre-clínicos. (A, B, C) presión proximal, flujo inspiratorio y volumen medio espirado (digitalizado de la figura 24 en Becker y Donn ${ }^{[26]}$, (D) Compliancia calculada según (2) para el neonato.

Continuando con el segundo paso del ensayo pre-clínico, según la Figura 9. En las Figuras 9.a y 9.b se observan los médicos en contacto con el pulmón de conejo y 3 de ellos ubicados en torno del neonato después de deslizar la cúpula a una posición similar a la mostrada en la Figura 2.b o Figura 2.d. En la figura 9.c se observa al pediatra preparando la conexión neumática en Y para el ensayo en un pulmón de conejo. En la figura 9.d se muestra un ejemplo de espiración en pulmón de conejo. En la figura 9.e se muestra un ejemplo exagerado de inspiración en pulmón de conejo, de manera que sea apreciable su visualización en el presente reporte. 


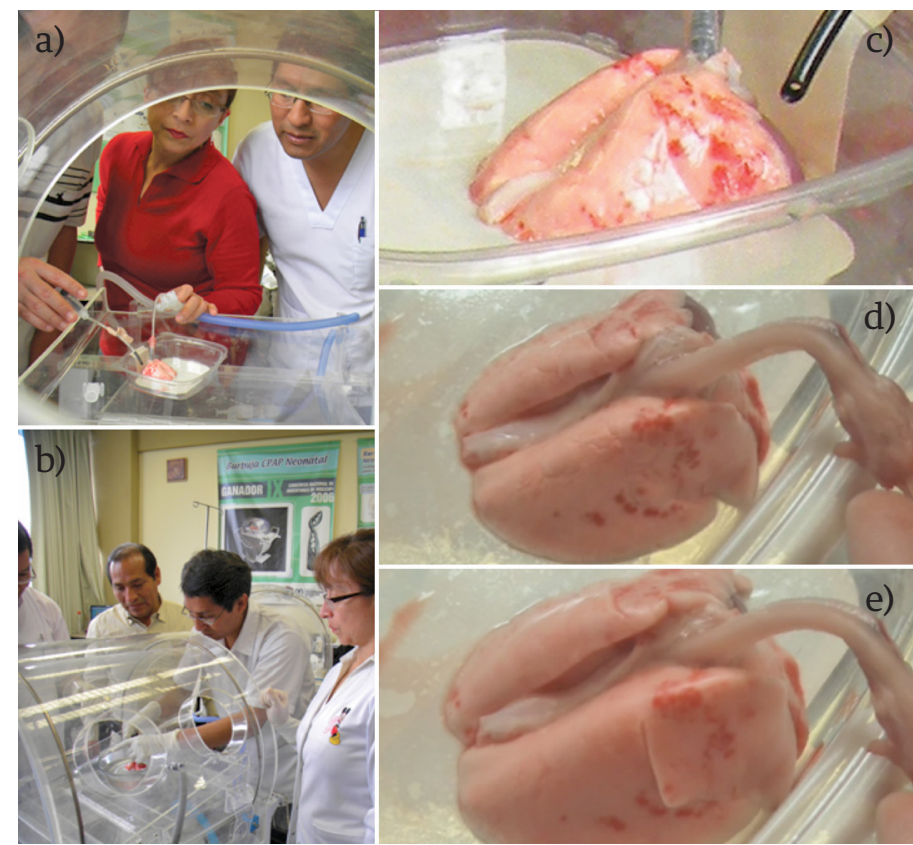

FIGURA 9. Ensayos pre-clínicos.

\section{(A, B) Pediatras y profesionales electrónicos preparando} el ensayo en un pulmón de conejo, (C) Preparando la conexión del pulmón de conejo a la tubería Y de ventilación pulmonar, (D) Espiración en pulmón de conejo, (E) Inspiración en pulmón de conejo.

Luego los pulmones de conejo fueron conectados a la pieza en "Y" del ventilador ESVIN, iniciándose el proceso de ventilación con los los parámetros obtenidos según se indica en la Tabla 2.

TABLA 2. Parámetros de ventilación mecánica preclínica obtenidos en ESVIN.

\begin{tabular}{|l|c|}
\hline \multicolumn{2}{|c|}{ Ventilación preclínica } \\
\hline $\begin{array}{l}\text { Presión inspiratoria } \\
\text { máxima (PIM) }\end{array}$ & $10 \mathrm{cmH} 2 \mathrm{O}$ \\
\hline Flujo & $2 \mathrm{LPM}$ \\
\hline $\begin{array}{l}\text { Frecuencia Respiratoria } \\
\text { (respiraciones/minuto) }\end{array}$ & 40 \\
\hline $\begin{array}{l}\text { Relación } \\
\text { Inspiración/Espiración }\end{array}$ & $1 / 2$ \\
\hline Temperatura & $30^{\circ} \mathrm{C}$ \\
\hline
\end{tabular}

En un cálculo aproximado: el cociente flujo de 2 LPM con frecuencia respiratoria de 40 respiraciones por minuto y el tiempo de inspiración de $0.5 \mathrm{~s}$ (relación de inspiración 1/2) da volumen de intercambio o tidal de $16.67 \mathrm{~mL}$; y la diferencia de presiones es de aproximadamente $10 \mathrm{cmH} 2 \mathrm{O}$ pico con la positiva menos la presión positiva final de la espiración de $1 \mathrm{cmH} 2 \mathrm{O}$, dando unos $9 \mathrm{cmH2O}$. Tomando estos datos tendríamos que en promedio la compliancia inspiratoria sería de 1.85 $\mathrm{mL} / \mathrm{cmH2O}$, lo cual está en el rango de compliancia neumática en neonatos mostrados en la Figura 8.

Cualitativamente: Al inicio no se evidencian mayores cambios en la expansión pulmonar, a medida que se va aumentando el volumen se originan cambios de presión y los pulmones se van distendiendo de menor a mayor magnitud, esto es lo que se conoce como distensibilidad estática la cual refleja las propiedades elásticas del pulmón. Cabe resaltar que cualquier obstrucción en la vía aérea, como sucedió en el ensayo (debido a la presencia de un coágulo en la tráquea) aumenta la resistencia y obstaculiza el paso de aire a través de la vía aérea. Esto se superó al aspirar el coágulo.

Se fue incrementando lentamente el volumen, produciendo expansión pulmonar y llegando a presiones pico de 15, 20, 25 y $30 \mathrm{cmH2O}$. Al llegar a $30 \mathrm{cmH} 2 \mathrm{O}$ se produce una fuga de aire en lóbulo apical izquierdo. Se intentó llegar a presiones más altas pero el ventilador mecánico en prueba tiene un mecanismo de seguridad que no permite llegar a niveles de PIM mayores de 30 cmH2O. Durante el proceso se notó una correlación cualitativamente adecuada entre presión administrada e insuflación pulmonar obtenida.

Todo el proceso fue cuidadosamente observado por 7 profesionales de Ingeniería electrónica, colaboradores del proyecto, dándole especial énfasis a las gráficas registradas en el monitor y también a la válvula de seguridad de presión, la cual se fue variando de parámetros para poner a prueba su eficacia. 


\section{CONCLUSIONES}

La presente realización de ESVIN comprobó su eficacia en las funciones de incubación con un tiempo de 52 minutos para estabilizar el interior del habitáculo y de 58 minutos para estabilizar la piel del neonato. Además, los estudios pre-clínicos en pulmones de conejos demuestran que es posible el control de inspiración y espiración (Figura 8) de un neonato con un pulmón de distensibilidad equivalente al rango de 26 a 32 semanas de gestación. Los ensayos pre-clínicos se realizaron dentro de los límites previstos, no ocurriendo ningún evento adverso o fuera de control. Sin embargo, queda pendiente medir experimentalmente la distensibilidad pulmonar, por la dificultad técnica de mantener los pulmones insuflados debajo del agua dentro de los recipientes graduados para tal efecto.

Debido a que en el experimento extirpamos el pulmón de la caja torácica y la consecuente pérdida de resistencia neumática, puede generar lesiones en los pulmones de conejos, sobre todo con presiones por encima de 30 cmH2O. Anteriormente se ha reportado efectos de la inspiración in vivo, donde de acuerdo al grado de presión se va incrementando el volumen alveolar, hasta llegar a una sobredistensión por volumen ${ }^{[27]}$. Sin embargo, desde nuestro experimento podemos decir no solo que es importante el grado de presión alcanzado (barotrauma), sino el grado de distensión pulmonar regional y de sobredistensión por volumen (volutrauma) lo que contribuye a la lesión pulmonar.

\section{DISCUSIÓN GENERAL}

El equipo de soporte de vida neonatal ofrece las siguientes ventajas técnicas:

1. Permite mayor fiabilidad del proceso de ventilación mecánica al neonato intubado, porque las tuberías de conexión entre el equipo y el neonato quedan fijas a la cuna, independientemente del movimiento del anillo térmico. Permite el rápido y amplio acceso al paciente para realizar procedi- mientos médicos de emergencia, tal como intubación endotraqueal, o cuando se está ventilando, sin requerir de un giro de $90^{\circ}$ al neonato sobre su cuna, e inclusive permite cirugías sin movilizar al neonato de la cuna; debido a que el anillo térmico que circunda a la superficie de la cuna se desliza longitudinalmente al habitáculo.

2. Uniformiza la temperatura dentro del habitáculo, mediante resistencias calefactoras que se encuentran en el espacio interior del anillo, las cuales calientan el flujo de aire que pasan alrededor de ellas. Tal como se demostró en la Figura 6, si bien la temperatura en el neonato demora en estabilizarse 6 minutos más que la del habitáculo, la temperatura en el neonato sería más estable, estabilizando con un rango de error de $0.17^{\circ} \mathrm{C}$. Esto tiene consecuencias favorable para el neonato, sabiendo que simulaciones han sugerido que la variación de temperatura dentro de una incubadora es de $0.6^{\circ} \mathrm{C}$ de acuerdo con Kim ${ }^{[28]}$, además variaciones de temperatura en el ambiente son importantes en la temperatura cerebral de cerdos neonatales de $14 \mathrm{~h}$ [29] y cobra más importancia dado que la relación cerebro/masa del cerdo neonatal es del $2 \%$ que es menor al $18 \%$ en el neonato humano que tendría implicancia en futuro desarrollo del neonato, según se dijo en la introducción. Finalmente, cerca del $45 \%$ de neonatos de menos de 32 semanas de gestación entre 26 y 32 semanas de nacidos en el Reino Unido tienen problemas cognitivos o de malformaciones cerebrales. Es decir, tal como se manifestó en la introducción creemos que un futuro uso de este equipo en neonatos mejorará la asistencia térmica y podría permitir permanecer menos tiempo en ventilación asistida y por consiguiente tener una mayor masa cerebral al largo plazo.

3. El equipo implementado evita la condensación del agua en las tuberías, mediante la ubicación de un segundo recipiente con agua (humidificador) den- 
tro del habitáculo y del tanque en el interior del anillo térmico, anulando las diferencias de temperaturas entre los procesos de ventilación, humidificación e incubación. Esta minimización de condensación al reducir la incomodidad del neonato puede reducir el tiempo de terapia ventilatoria y prevenir la elongación de ciertos marcadores bioleléctricos como el retraso de las P300 mostrado en niños en el caso de problemas ventilatorios ${ }^{[30]}$.

4. Reduce la resistencia al flujo de gases de la línea de inspiración, debido a que la línea de inspiración es mucho más corta al ubicar el humidificador cerca al neonato. En nuestra ESVIN, la ventilación y la calefacción están integradas y esto hace que las longitudes del conducto que va desde el humidificador hacia el neonato y del neonato hacia la válvula de espiración son de 60 $\mathrm{cm}$ y $60 \mathrm{~cm}$, respectivamente, con diámetros de $12.7 \mathrm{~mm}$. En contraparte en la práctica usual, debido al uso complementario de equipos, las distancias son de $150 \mathrm{~cm}$ (e.g. complementación empleada por las NICU en el país), respectivamente, creando una resistencia aproximada de $2.5 \mathrm{cmH} 2 \mathrm{O}$ en las mismas condiciones de manejo y diámetros de las tuberías.
5. Puede trabajar en ambientes abiertos, debido al principio del anillo. En el caso del presente prototipo el anillo es doble (Figura 1.a) y hay un anillo que se ubicó dentro del prototipo (Figura 3.a izquierda) y otro hacia la parte externa por donde se desliza la cúpula (Figura 3.b derecha), esto permite seguir irradiando calor de forma ininterrumpida y sin movilizar al neonato. Si bien al abrir la parte lateral de la cúpula se expone la cabeza del neonato a la temperatura ambiente, no tendría desventaja frente a los modelos de NICU, pues habría otras fuentes de calor por parte del personal médico, que normalmente obstruyen la fuente de calor de las NICU (e.g. Koch en ${ }^{[9]}$ ).

\section{AGRADECIMIENTOS}

Se agradece al Programa Nacional de Innovación para la Competitividad y Productividad, Innóvate Perú del Ministerio de la Producción por financiar el Proyecto "Incubadora con Respirador Artificial" (2016-2018).

Así como al Unidad Coordinadora del Programa de Ciencia y Tecnología de la Presidencia del Consejo de Ministros (FINCYT) por financiar el proyecto "Equipo Telemédico de Soporte de Vida para Neonatos Críticos” (2009-2011). 


\section{REFERENCIAS}

[1] Mugruza-Vassallo CA. Análisis, diseño y prueba de transductores de flujo de gases. Disertación de tesis. Pontificia Universidad Católica del Perú; 2001

[2] Castillón-Lévano B, Ajito-Lam E, inventores; Pontificia Universidad Católica del Perú, titular. Neonatal Artificial Bubble US6884211B2. 2005, Apr 26.

[3] Castillón-Lévano B, Ajito-Lam E, inventores; Pontificia Universidad Católica del Perú, titular. Neonatal Artificial Bubble EP1380276B1. 2007, Mar 28.

[4] Castillón-Lévano B, inventor; Pontificia Universidad Católica del Perú, titular. Method to linearly measure gas volume flow in ducts and flow sensor US7028560. 2006, Apr 18

[5] Castillón-Lévano B, Ajito-Lam E, Albino-Rivera CA, BarriosMontalvo AR, Chipana-Quispe RD, Davalos-Pinto JA, ObregonNuñez JM, Quispe-Salcedo RN, Solorzano Rosales-Edwin M, Tarrillo-Olano JF, Yamamoto-Noguchi CC, inventores; Pontificia Universidad Católica del Perú, titular. Burbuja Neonatal con Presurizador de Vías Aéreas. PE001567-2006/OIN. 2012, Ene 26

[6] Bowden K, Laswick R, inventor; O two Medical Technologies Inc, titular. Automatic ventilator for cardio pulmonary resucitation with chest compression timer and ventilation alarms. EP1529547. 2005, May 11.

[7] Eger M, Matthiessen H, Weismann D, inventores; Draeger Medical $\mathrm{GmbH}$, titular; Vorrichtung und Versorgen eines Patienten mit Atemgas und Verfahren Vorrichtung zum Regeln einer BeatmungsVorrichtung. DE102006030520. 2007, Jun 21

[8] Otte, T; Draeger Medical GmbH. Incubator with diferent operating states”. US2008125619. 2008, May 29 y US8613697B2. 2013, Dic 24

[9] Otte T, Koch J, Chung-Che A, inventores; Draeger Medical GmbH. Incubator or open patient care unit with automatically actuated side panes”. US2008081943. 2008, Apr 03 y US8613697B2. 2013, Dic 24

[10] Miyagawa K, Sato M, Matsubara K, inventores; Atom Medical Corp, titular. Oxygen concentration control apparatus for incubator, and incubator using the same" US2008076962. 2008, Mar 27

[11] Merritt TA, Pillers D, Prows SL. Early NICU discharge of very low birth weight infants: a critical review and analysis. InSeminars in neonatology 2003 Apr 1 (Vol. 8, No. 2, pp. 95-115). Elsevier. DOI: https://doi.org/10.1016/S1084-2756(02)00219-1

[12] Chaikind $\mathrm{S}$, Corman $\mathrm{H}$. The impact of low birthweight on special education costs. Journal of health economics. 1991 Oct 1;10(3):291311. DOI: https://doi.org/10.1016/0167-6296(91)90031-H

[13] Anderson PJ, Hunt RW, Lee KJ, Doyle LW, Inder TE. Neurodevelopmental and perinatal correlates of simple brain metrics in very preterm infants. Archives of pediatrics \& adolescent medicine. 2011 Mar 7;165(3):216-22. DOl: https://doi.org/10.1001/ archpediatrics.2011.9

[14] Castillón-Lévano CB, Mugruza-Vassallo CA, Coello-Durán JL, inventores; Pontificia Universidad Católica del Perú, titular. Neonatal Intensive Care Unit. PCT/IB2009-007014. 2009, Sep 11 y WO/2011/030177. 2011, Mar 17
[15] International Electrotechnical Commission. This particular standard amends and supplements IEC 60601-1:2005, Medical electrical equipment - Part 1: General requirements for basic safety and essential performance. This second edition cancels and replaces the first edition published in 1990 and its Amendment 1 (1996). This edition constitutes a technical revision. It was revised to structurally align with the third edition (2005) of IEC 60601-1. IEC 60601-2-19:2009 (2009) specifies safety requirements for infant incubators.

[16] International Electrotechnical Commission. Amendment 1 Medical electrical equipment - Part 1: General requirements for safety - 4. Collateral standard: Programmable electrical medical systems. IEC 60601-1-4-am1 ed1.0. 1999, Oct.

[17] International Electrotechnical Commission. Medical electrical equipment - Part 2-12: Particular requirements for the safety of lung ventilators - Critical care ventilators. IEC 60601-2-12 ed2.0 withdrawn. 2001, Oct 24.

[18] Forsberg $\mathrm{K}, \mathrm{Mooz} \mathrm{H}$. The relationship of system engineering to the project cycle. InINCOSE International Symposium 1991 Oct 1 (Vol. 1, No. 1, pp. 57-65). DOI: https://doi. org/10.1002/j.2334-5837.1991.tb01484.x

[19] Forsberg K, Mooz H, Cotterman H. Visualizing Project Management, $3^{\mathrm{a}}$ edición, John Wiley and Sons, Nueva York, NY, 2005.

[20] Mugruza-Vassallo C. Van der Waals and Ideal gas models for compressibility by means of pressure in pneumatic pipes from 1 to 100 Lpm. InEngineering in Medicine and Biology Society, 2004. IEMBS'04. 26th Annual International Conference of the IEEE 2004 Sep 1 (Vol. 1, pp. 2034-2037). IEEE. DOI: https://doi.org/10.1109/ IEMBS.2004.1403598

[21] TE Connectivity. Sensores de temperatura [Internet] (Accesado el 2010, Jun 12). Disponible en: https://goo.gl/SHpeq9

[22] Honeywell Inc. Sensores de Presión [Internet] 40PC001B1A (Accesado el 2010, Jun 12). Disponible en: https://goo.gl/XYxoMz

[23] Honeywell Inc. Sensores de flujo [Internet] AWM5104VA (Accesado el 2010, Jun 12). Disponible en: https://goo.gl/kLKVzA

[24] Laubscher TP, Heinrichs W, Weiler N, Hartmann G, Brunner JX. An adaptive lung ventilation controller. IEEE Transactions on Biomedical Engineering. 1994 Jan;41(1):51-9. DOI: https://doi. org/10.1109/10.277271

[25] Mugruza-Vassallo C. Flow gas transducer on basis of a hot wire with a nickel-titanium alloy in its flow calibration system. InEngineering in Medicine and Biology Society, 2001. Proceedings of the 23rd Annual International Conference of the IEEE 2001 (Vol. 3, pp. 3124-3127). IEEE. DOI: https://doi.org/10.1109/ IEMBS. 2001.1017464

[26] Becker MA, Donn SM. Real-time pulmonary graphic monitoring. Clinics in perinatology. 2007 Mar 1;34(1):1-7. DOl: https://doi. org/10.1016/i.clp.2006.12.002 
[27] Tsuchida S, Engelberts D, Peltekova V, Hopkins N, Frndova H, Babyn P, McKerlie C, Post M, McLoughlin P, Kavanagh BP. Atelectasis causes alveolar injury in nonatelectatic lung regions. American journal of respiratory and critical care medicine. 2006 Aug 1;174(3):279-89. DOI: https://doi.org/10.1164/rccm.200506$10060 \mathrm{C}$

[28] Kim YH, Kwon CH, Yoo SC. Experimental and numerical studies on convective heat transfer in a neonatal incubator. Medical and Biological Engineering and Computing. 2002 Jan 1;40(1):114-21. DOI: https://doi.org/10.1007/BF02347704
[29] Liu X, Chakkarapani E, Hoque N, Thoresen M. Environmental cooling of the newborn pig brain during whole body cooling. Acta Paediatrica. 2011 Jan 1;100(1):29-35. DOl: 10.1111/j.1651-2227.2010.01956.x

[30] Guan GT, Jin YP, Zheng RP, Liu FQ, Wang YL. Cognitive P300 evoked potentials in school age children after surgical or transcatheter intervention for ventricular septal defect. Pediatrics International. 2011 Dec 1;53(6):995-1001. DOI: 10.1111/j.1442-200X.2011.03407.x 